\title{
Research on Energy Efficiency vs. Cooperative BSs' Number of Coordinated Multi-Point Transmission
}

\author{
http://dx.doi.org/10.3991/ijoe.v12i06.5523 \\ Shuai Liu, Jianxin Dai, Jie Qi \\ Nanjing University of Posts and Telecommunications, Nanjing, Jiangsu, China
}

\begin{abstract}
This paper focus on energy efficiency (EE) in Coordinated Multi-Point (CoMP) transmission with perfect feedback under single-user scenario. Considering the power consumed by cooperative BSs, an energy-efficient optimization function is established. This optimization goal is simplified after analyzing capacity of non-CoMP and CoMP. Then the relationship between energy efficiency and cooperative BSs' number is analyzed. Simulation results show that when the selected number of cooperative BSs is smaller than a threshold, EE increases with the increasing of the number of cooperative BSs and when it exceeds the threshold, EE decreases with the increasing of the number of cooperative BSs.
\end{abstract}

Index Terms-Coordinated Multi-Point;Energy-Efficient; Green communications

\section{INTRODUCTION}

In many practical systems, SINR is low, especially near the cell edge. Coordinated Multi-Point (CoMP) transmission technology is proposed to solve this issue by $3 \mathrm{GPP}$ [1].

There are two main schemes available for CoMP transmission: Joint Processing (JP) and Coordinated scheduling/beamforming (CS/BF).JT-CoMP, in LTEAdvanced HetNets under unreliable backhaul network is investigated in [2]. The user throughput and spectral efficiency of CoMP is analyzed in[[3]. Similarly, a downlink transmission mode selection method is proposed in [4].

However, one of the main challenges in CoMP transmission is energy consumption as several information between coordinated BSs need to be exchanged[5]. The detailed survey on energy efficient CoMP has been discussed in [6]-[8].

A energy-efficient design for heterogeneous network (HetNet) CoMP architecture is proposed [6]. A scheme to maximize the minimum weighted energy efficiency (EE) with QoS constraint is given in [7]. A approach of energy efficient CoMP precoding is designed in HetNets [[8].Enhanced Multimedia Broadcast Multicast Service (E-MBSFN), as a multi-cell transmission system, introduces a single frequency network transmission, namely realizing synchronized transmission using the same block of time and frequency in multi-cells[9].

In this paper, differently from other recent papers, the relationship between energy efficiency (EE) and cooperative

BSs'number is explored in this paper. Considering the power consumed by cooperative BSs, an energy efficiency (EE) function is derived. Then the issue about EE vs. cooperative BSs'number is investigated by means of mathematical analysis.

The paper is organized as follows. In Section II, the system model is introduced. Section III formulates the problem and proposes the solutions. In section IV, simulation results are shown and analyzed. The paper is concluded in Section V.

Notations: $\|(\cdot)\|_{2},\|(\cdot)\|_{F}$ denote the spectral norm and the Frobenius norm respectively. The transpose, the conjugate (Hermitian) transpose are written as $(\cdot)^{T}$ and $(\cdot)^{H}$ respectively. $\operatorname{diag}\left(a_{1}, \mathrm{~L}, a_{k}\right)$ is a diagonal matrix with elements $a_{1}, \mathrm{~L}, a_{k}$ on the main diagonal. $E\{\cdot\}$ is the expectation operator

\section{SYSTEM MODEL}

\section{A. System model based on the single-user scenario}

The system model is based on the single-user scenario. The model of the multi-user scenarios is easily obtained by generalizing the single-user scenario. On the principle of cooperative cell clustering and for single cluster collaboration model, a typical double-cell cellular collaboration system composed by seven hexagonal cell is considered. The single-user downlink model is shown in Figure 1. The cell edge user UE simultaneously receives the transmission signal from the serving cell Cell 0 and two cooperative cells i.e. Cell 1 and Cell 2 . The transmission signals of the remaining cells are interference sources. While the cell center user UE only receives the transmission signal from Cell 0.

Figure 1 shows the system model based on the singleuser scenario. For the network consisting of seven cells, i.e., the base station number $M$ is 7 ,and each base station is configured with $N_{t}$ transmitting antennas, and the mobile users are distributed randomly and uniformly in each cell. Each base station can select several base stations to collaborate with. Furthermore, the user is equipped with a single receiving antenna, that is $N_{r}=1$.

For the primary cell user, the received signal is

$$
y=\mathbf{h}_{0} \mathbf{w}_{0}^{H} x_{0}+\sum_{m=1}^{6} \mathbf{h}_{m} \mathbf{w}_{m}^{H} x_{m}+n
$$

where, $x_{m}$ denotes the downlink single-stream data; $\mathbf{h}_{m}$ is the channel vector from the base station to this user; 
$\mathbf{w}_{m}^{H}$ is the precoding matrix from the base station to the user, and $n$ is the additive Gaussian white noise with the mean of 0 and variance of 1 .

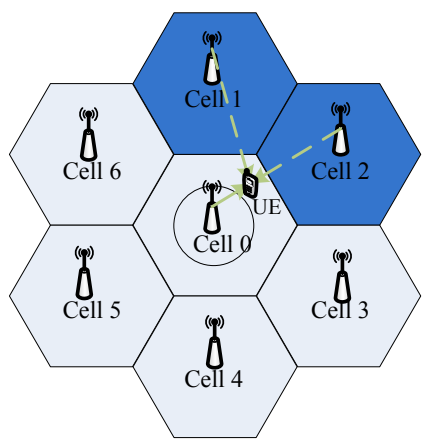

the serving cell signal $\longrightarrow$
the cooperative cell signal $\longrightarrow \rightarrow$

Figure 1. The model of CoMP based on the single-user scenario

\section{B. Ergodic Capacity}

When the user without using CoMP technology is in the center region, the Ergodic capacity of non-CoMP $\bar{C}^{\mathrm{N}}$ can be organized into

$$
\bar{C}^{\mathrm{N}}=\mathrm{E}_{\mathbf{h}}\left[\log _{2}\left(1+\frac{E\left\{\left\|\mathbf{h}_{0} \mathbf{w}_{0}^{H} x_{0}\right\|_{F}^{2}\right\}}{E\left\{\left\|\sum_{m=1}^{6} \mathbf{h}_{m} \mathbf{w}_{m}^{H} x_{m}\right\|_{F}^{2}\right\}+\sigma^{2}}\right)\right]
$$

Here, $Y$ is defined as the cooperative set of the user; the interfering set is $\mathrm{Y}$; the size of $\mathrm{Y}$ is referred to as $M_{C}$, which means the number of the cooperated base station. Then the Ergodic capacity of CoMP $\bar{C}^{\mathrm{C}}$ can be organized into can be defined as

$$
\bar{C}^{\mathrm{C}}=\mathrm{E}_{\mathbf{h}}\left[\log _{2}\left(1+\frac{E\left\{\left\|\mathbf{H} \mathbf{w} x_{0}\right\|_{F}^{2}\right\}}{E\left\{\left\|\sum_{m \in \Psi} \mathbf{h}_{m^{\prime}} \mathbf{w}_{m^{\prime}}^{H} x_{m^{\prime}}\right\|_{F}^{2}\right\}+\sigma^{2}}\right)\right]
$$

where $\mathbf{H}=\left[\mathbf{h}_{0}, \mathbf{h}_{1}, \mathrm{~L}, \mathbf{h}_{M_{c}}\right], \mathbf{w}=\left[\mathbf{w}_{0}^{H}, \mathbf{w}_{1}^{H}, \mathrm{~L}, \mathbf{w}_{M_{c}}^{H}\right]^{T}$.

The solution to this problem is as follow: First, a radius $(r, q)$ is provided to draw the boundaries of CoMP and non-CoMP area. Then, in the CoMP area we select the cooperative BSs by the principle of proximity.

Due to cellular system with good symmetry and assuming all users and base stations are distributed uniformly, the approximately probability density function for $(r, q)$

is $\quad f(\rho, \theta) \approx \frac{2 \sqrt{3}}{9 R^{2}} \rho, 0 \leq \rho \leq R, 0 \leq \theta \leq 2 \pi$

Ergodic capacity of the user in the cell is given below considering the hexagonal symmetry

$$
\bar{C}_{\text {erg }}=6 \int_{-\frac{\pi}{6}}^{\frac{\pi}{6}} \int_{0}^{r} \rho f(\rho, \theta) \bar{C}^{\mathrm{N}} \mathrm{d} \rho \mathrm{d} \theta+6 \int_{-\frac{\pi}{6}}^{\frac{\pi}{6}} \int_{r}^{\frac{\sqrt{3} R}{2 \cos \theta}} \rho f(\rho, \theta) \bar{C}^{\mathrm{C}} \mathrm{d} \rho \mathrm{d} \theta
$$

where $\bar{C}^{\mathrm{N}}, \bar{C}^{\mathrm{C}}$ show the subscriber capacity located at the region of non-CoMP and CoMP respectively.

\section{Power Consumption}

The total power consumed by the CoMP system includes two parts: one part is counted as circuit er $P_{C}$; The other power is consumed by the cooperative stations to exchange information. Then, the total power can be expressed as

$$
P_{\text {total }}=M_{C} P_{0}+P_{C}
$$

Where $M_{C}$ represents the number of cooperative stations in this model.

\section{Energy-efficient Mathematical model}

Energy-efficient CoMP model is given by

$$
\max _{r, M_{C}} E E=\max _{r, M_{C}} \frac{\bar{C}_{\mathrm{erg}}}{\left(M_{C} P_{0}+P_{C}\right)^{k}}
$$

Where $k$ is a positive number, acts as a weight factor, (2)which is independent of the other parameters.Here, a new function $\varphi\left(M_{C}, r, \lambda\right)$ is defined as

$$
\varphi\left(M_{C}, r, \lambda\right)=\bar{C}_{\mathrm{erg}}-\lambda\left(M_{C} P_{0}+P_{C}\right)^{k}
$$

Where $\lambda$ is a positive number.Then the optimization problem can be converted as

$$
\begin{aligned}
& \max _{r, M_{c}} E E \approx \max _{r, M_{c}} \varphi\left(M_{C}, r, \lambda\right)=\bar{C}_{\mathrm{erg}}-\lambda\left(M_{C} P_{0}+P_{C}\right)^{k} \\
& \text { s.t. }\left\{\begin{array}{l}
0<r<\sqrt{3} R / 2 \\
\bar{C}^{\mathrm{N}}, \bar{C}^{\mathrm{C}} \\
\lambda>0 \\
k \geq 1, k \in N^{*}
\end{array}\right.
\end{aligned}
$$

\section{THEORETICAL ANALYSIS}

When the user is in the service cell-center area, i.e. $(\rho, \theta) \in S_{\mathrm{N}}$, the ergodic capacity of non-CoMP is

$$
\bar{C}^{\mathrm{N}}=\mathrm{E}_{\mathbf{h}}\left[\log _{2}\left(1+\frac{E\left\{\left\|\mathbf{h}_{0} \mathbf{w}_{0}^{H} x_{0}\right\|_{F}^{2}\right\}}{E\left\{\left\|\sum_{m=1}^{6} \mathbf{h}_{m} \mathbf{w}_{m}^{H} x_{m}\right\|_{F}^{2}\right\}+\sigma^{2}}\right)\right]
$$

In this mode, precoding vector $\mathbf{w}_{m}^{H}(m=0, \mathrm{~L}, 6)$ is random variable independent of channel information as well 
as data information, and obeys independent complex Gaussian distribution, and $\mathrm{E}\left\{\left\|\mathbf{w}_{m^{\prime}}^{H}\right\|_{F}^{2}\right\}=P_{m}$, where $P_{m}$ is the transmitted power of the $m$ th base station. Therefore, we get

$$
\begin{aligned}
& E\left\{\left\|\mathbf{h}_{0} \mathbf{w}_{0}^{H} x_{0}\right\|_{F}^{2}\right\}=P_{0}\left\|\mathbf{h}_{0}\right\|_{F}^{2}, \\
& E\left\{\left\|\sum_{m=1}^{6} \mathbf{h}_{m} \mathbf{w}_{m}^{H} x_{m}\right\|_{F}^{2}\right\}=\sum_{m=1}^{6} P_{m}\left\|\mathbf{h}_{m}\right\|_{F}^{2}
\end{aligned}
$$

Without considering shadow fading, the channel fading variable is denoted by $m$ th $\mathbf{h}_{m}=l_{m} \mathbf{f}_{m}$, where $l_{m}$ is for the large scale fading and $\mathbf{f}_{m}$ is fast fading random vectors that obeys complex Gaussian distribution.

$$
\bar{C}^{\mathrm{N}}=\mathrm{E}_{\mathbf{f}}\left[\log _{2}\left(1+\frac{P_{0} L_{0}\left\|\mathbf{f}_{0}\right\|_{F}^{2}}{\sum_{m=1}^{6} P_{m} L_{m}\left\|\mathbf{f}_{m}\right\|_{F}^{2}+\sigma^{2}}\right)\right]
$$

where, $L_{m}=l_{m}^{2}$ represents power loss factor generated by path loss.

When a user is at the edge of the cell, it chooses the nearest coordinated base stations to send downlink data. The remaining cells are non-cooperative cells, and the user receives a signal from them as the interference signal, which is denoted by

$$
y_{\text {Int }}^{\mathrm{C}}=\sum_{m^{\prime} \in \bar{\Psi}} \mathbf{h}_{m^{\prime}} \mathbf{w}_{m^{\prime}}^{H} x_{m^{\prime}}
$$

The precoding vector and transmission signal are independent random vectors, i.e.

$$
\begin{aligned}
& \mathrm{E}_{\mathbf{w}_{m^{\prime}}^{H}}\left\{\left\|y_{\text {Int }}^{\mathrm{C}}\right\|_{F}^{2}\right\}=\mathrm{E}\left\{\left\|\sum_{m^{\prime} \in \bar{\Psi}} \mathbf{h}_{m^{\prime}} \mathbf{w}_{m^{\prime}}^{H} x_{m}\right\|_{F}^{2}\right\} \\
& =\sum_{m^{\prime} \in \bar{\Psi}} \mathrm{E}\left\{\left\|\mathbf{h}_{m^{\prime}} \mathbf{w}_{m^{\prime}}^{H}\right\|_{F}^{2}\right\}=\sum_{m^{\prime} \in \bar{\Psi}} \mathbf{h}_{m^{\prime}} \mathrm{E}\left\{\left\|\mathbf{w}_{m^{\prime}}^{H}\right\|_{F}^{2}\right\} \mathbf{h}_{m^{\prime}}^{H}
\end{aligned}
$$

For non-cooperative cell, let $\mathrm{E}\left\{\left\|\mathbf{w}_{m^{\prime}}^{H}\right\|_{F}^{2}\right\}=1, \mathbf{h}_{m^{\prime}}=l_{m^{\prime}} \mathbf{f}_{m^{\prime}}$

$$
\begin{aligned}
& \mathrm{E}_{\mathbf{f}_{m^{\prime}}} \mathrm{E}_{\mathbf{w}_{m^{\prime}}^{H}}\left\{\left\|y_{\mathrm{Int}}^{\mathrm{C}}\right\|_{F}^{2}\right\} \\
& =\sum_{m^{\prime} \in \bar{\Psi}} \mathrm{E}_{\mathbf{f}_{m^{\prime}}}\left\{\mathbf{h}_{m^{\prime}} \mathbf{h}_{m^{\prime}}^{H}\right\}=\sum_{m^{\prime} \in \bar{\Psi}} l_{m^{2}}^{2} \mathrm{E}_{\mathbf{f}_{m^{\prime}}}\left\{\mathbf{f}_{m^{\prime}} \mathbf{f}_{m^{\prime}}^{H}\right\}=\sum_{m^{\prime} \in \bar{\Psi}} N_{t} l_{m^{\prime}}^{2}
\end{aligned}
$$

Based on the assumption that the power allocation on each antenna are equal, the non-cooperative cell interference is

$$
I=\sum_{m^{\prime} \in \bar{\Psi}} \frac{P_{m^{\prime}}}{N_{t}} N_{t} l_{m^{\prime}}^{2}=\sum_{m^{\prime} \in \Psi} P_{m^{\prime}} l_{m^{\prime}}^{2}
$$

The state ergodic capacity of the edge user is

$$
\bar{C}^{\mathrm{C}}=\mathrm{E}_{\mathbf{h}}\left[\log _{2}\left(1+\frac{\|\mathbf{H w}\|_{F}^{2}}{\sum_{m^{\prime} \in \bar{\Psi}} P_{m^{\prime}} l_{m^{\prime}}^{2}+\sigma^{2}}\right)\right]
$$

Then, the second derivative of $E E$ can be obtained as follows

$$
I=\sum_{m^{\prime} \in \Psi} \frac{P_{m^{\prime}}}{N_{t}} N_{t} l_{m^{\prime}}^{2}=\sum_{m^{\prime} \in \Psi} P_{m^{\prime}} l_{m^{\prime}}^{2}
$$

Where, $I=\sum_{m^{\prime} \in \Psi} \frac{P_{m^{\prime}}}{N_{t}} N_{t} l_{m^{\prime}}^{2}=\sum_{m^{\prime} \in \bar{\Psi}} P_{m^{\prime}} l_{m^{\prime}}^{2}$.

Obviously, the secondary derivation result is negative, i.e. $\partial E E / \partial M_{C}$ decreasing from positive to negative. $r$ has an impact on decreasing speed. The physical meaning of the formula is as follows. When the selected number of cooperative BSs is smaller than a threshold, $E E$ increases with the increasing of $M_{C}$; moreover, when it exceeds the threshold, $E E$ decreases with the increasing of $M_{C}$. The changing speed is decided by $r$.

The value $r$ represents the cell edge region. If the edge region is close to the serving $\mathrm{BS}$, it will result in that the user do not need to cooperate. If the edge region is far from the serving BS, it results in that the user can not cooperate and the interference from neighbor BS is strong enough to make the user performance degraded. Therefore, the value cannot be too large or too small.

\section{NUMERICAL RESUlTS}

TABLE I.

SIMULATION SCENARIO AND PARAMETER

\begin{tabular}{|l|l|}
\hline Scenario & value \\
\hline Channel model & COST231 Hata Model \\
\hline Cell Radius & $1000 \mathrm{~m}$ \\
\hline standard deviation of shadowing & $8 \mathrm{~dB}$ \\
\hline Antenna Gain & $10 \mathrm{~dB}$ \\
\hline Carrier frequency & $1.9 \mathrm{GHz}$ \\
\hline Channel bandwidth & $20 \mathrm{MHz}$ \\
\hline Path loss factor & -3.7 \\
\hline
\end{tabular}

The system simulation scenario and parameters are shown as above.

According to COST231 Hata model, the path loss model is

$$
\begin{aligned}
& P L[\mathrm{~dB}]=\left(44.9-6.55 \log _{10}\left(h_{b s}\right)\right) \log _{10}\left(\frac{d}{1000}\right)+45.5+ \\
& \left(35.46-1.1 h_{m s}\right) \log _{10}\left(f_{c}\right)-13.82 \log _{10}\left(h_{b s}\right)+0.7 h_{m s}+C
\end{aligned}
$$

where, $h_{b s}, h_{m s}$ are heights of BS's and MS's antenna; $d$ is the distance between the BS and MS; Parameters for urban macrocells are. $h_{b s}=32 \mathrm{~m}, h_{m s}=1.5 \mathrm{~m}, f_{c}=1900 \mathrm{MHz}, C=3 \mathrm{~dB}$

The correction model of the path loss is

$$
P L=34.5+35 \log _{10}(d), \quad d \geq 35 \mathrm{~m} .
$$




\section{A. The Relationship between energy efficiency and different coordinated BSs with $k=1$.}

According to Figure 2, we can easily get a conclusion: with the cell radius of $1000 \mathrm{~m}$, the user does not need to select any BS to cooperate with within $300 \mathrm{~m}$ from the cell center; it needs to choose one BS within around $300 \mathrm{~m}$ to $500 \mathrm{~m}$ from the center, select two BSs within around $500 \mathrm{~m}$ to $650 \mathrm{~m}$ from the center, select three BSs within around $650 \mathrm{~m}$ to $750 \mathrm{~m}$ from the center and select four BSs as distance beyond $750 \mathrm{~m}$. If the edge region is close to the serving BS, it will result in that the user do not need to cooperate, which increases complexity, therefore, the energy efficiency of CoMP scheme is not high.

B. The Relationship between energy efficiency and different radius of user location with $k=1$.

Figure 3 shows that the energy efficiency of CoMP has a certain relationship with $M_{C}$. When the selected number of cooperative BSs is smaller than a threshold, $E E$ increases with the increasing of $M_{C}$; moreover, when it exceeds the threshold, $E E$ decreases with the increasing of $M_{C}$.

\section{CONCLUSIONS}

This paper addresses the energy efficiency analysis of CoMP transmission. The energy efficiency (EE) optimization problem is derived and simplified. We can draw conclusions from the above facts that the energy efficiency of CoMP has a certain relationship both with cooperative BSs' number and cooperative radius. Moreover different weight factor will bring in different energy efficiency when the user is in the same position.

\section{REFERENCES}

[1] 3GPP TR36.913, "Requirements for Further Advancements for EUTRA

[2] Technology Division, Grameenphone Limited. "Performance analysis of JT-CoMP transmission In Heterogeneous Network over unreliable backhaul" ICEEICT, 2015 International Conference on.pp.1-5

[3] Liew Jiun Terng ; Fac. of Eng." Performance analysis of Coordinated MultiPoint (CoMP) in long term evolution- advanced".Telecommunication Technologies (ISTT), 2014 IEEE 2nd International Symposium on,pp.379-384.

[4] Zhang, Q, Yang, C. "Transmission Mode Selection in Cooperative Multi-cell Systems Considering Training Overhead". In Proceedings 2011 IEEE 73rd Vehicular technology conference, pp. 1-5, Beijing (China). http://dx.doi.org/10.1109/vetecs.2011.5956634

[5] Jianxin Dai,SHuai Liu."Robust Design of Coordinated Set Planning with the Non-Ideal Channel".KSII Transactions On Internet And Information Systems.,2014,pp.1654-1673

[6] Saidul Huq, K.M. Inst. de Telecomun., Aveiro, Portugal Mumtaz, S. ; Rodriguez, J. ; Verikoukis, C."Investigation on Energy Efficiency in HetNet CoMP Architecture" Communications (ICC), 2014 IEEE International Conference on.pp. $1112-1117$.

[7] Bo Du Nat. Mobile Commun. Res. Lab., Southeast Univ., Nanjing, China Cunhua Pan;Wence Zhang;Ming Chen” Distributed Energy-Efficient Power Optimization for CoMP Systems With Max-Min Fairness" Communications Letters, IEEE ,18(6), 2014, pp.199-1002

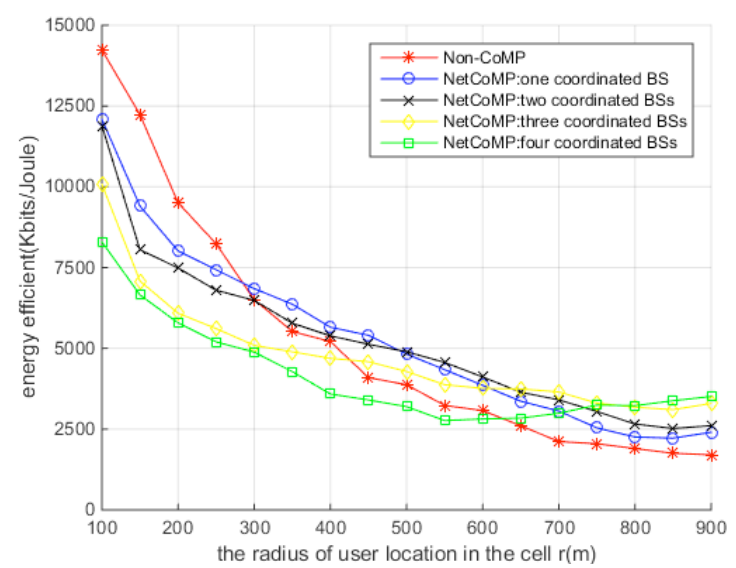

Figure 2. the comparison of energy efficiency for different coordinated BSs with $k=1$.

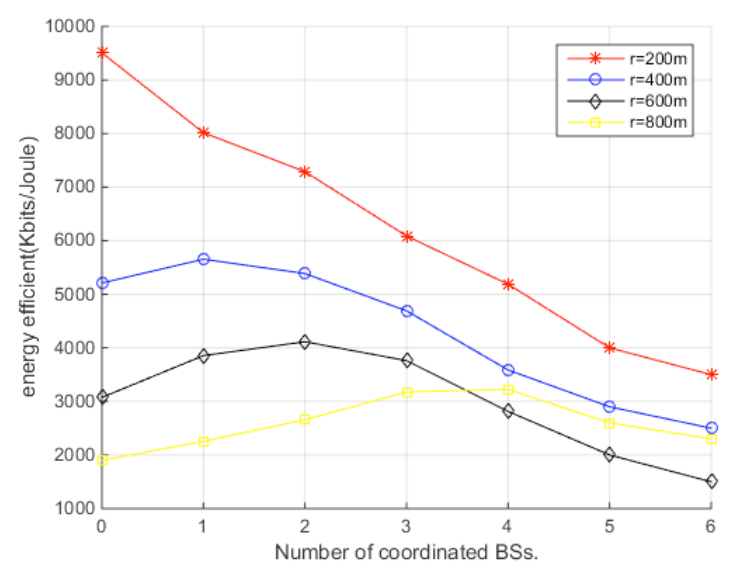

Figure 3. The comparison of energy efficiency for different radius of user location with $k=1$.

[8] ZhikunXu Sch. of Electron. \& Inf. Eng., Beihang Univ., Beijing, China Chenyang Yang;Li, G.Ye. ; Yalin Liu;Shugong Xu.” Energy-Efficient CoMP Precoding in Heterogeneous Networks" .62 (4 ),2014,pp.1005-1017

[9] Gomes P., Seguro J., \& Correia A. "MIMO Coordination for EMBMS Transmissions in LTE-A". In Proceedings 2010 IEEE 6th International Conference on Wireless and Mobile Communications, pp. 291-296, Valencia. http://dx.doi.org/10.1109/ icwmc. 2010.58

\section{AUTHORS}

Shuai Liu is with College of Telecommunications \& Information Engineering, Nanjing University of Posts and Telecommunications, Nanjing 210023, China (e-mail: liu977803265@163.com).

Jianxin Dai is with School of Science, Nanjing University of Posts and Telecommunications, Nanjing, 210023, China (e-mail: daijx@njupt.edu.cn)

Jie Qi is with College of Telecommunications \& Information Engineering, Nanjing University of Posts and Telecommunications, Nanjing 210023, China (e-mail: qijieaka@163.com).

This work is supported by STITP of Nanjing University of Posts and Telecommunications (No.SZD2015017), Postdoctoral Research funding plan in Jiangsu province (No.1501073B) and Natural Science Foundation of Nanjing University of Posts and Telecommunications (NY214108). Submitted 27 January 2016. Published as resubmitted by the authors 24 March 2016. 